\title{
Generation Dispatch Considering Wind Energy and System Reliability
}

\author{
K. Liu, Student Member, IEEE, J. Zhong Member, IEEE
}

\begin{abstract}
This paper attempts to address the issues of integrating wind generations with bulk power system while maintaining the efficiency and reliability of system operation. The stochastic output of wind generations increases the difficulty of balance total supply and load in a timely manner, and violates the system reliability indices such as EENS and LOLP. An efficient mean is to increase the operating reserve to compensate this additional unpredicted imbalance. To solve this problem, ideally the wind power, reserve and reliability cost should be concerned and optimized simultaneously. However the current dispatch and planning models with wind energy are mostly stochastic and solved by Monte Carlo simulation or heuristic methods. Those models and methods may not satisfy the requirements for mid-term and short term system operations, and further on-line applications. In this paper we propose an analytical EENS and LOLP indices contributed by wind power uncertainties with application of Q-function approximation. These reliability indices are considered in the co-optimization model of energy market and reserve market. In the model, conventional units and wind units are dispatched with optimal reserve and reliability costs. The wind power incurred system operating costs are proposed and formulated by the sensitivities in the optimization model. Finally the numerical example based on IEEE-39 system shows validity and effectiveness of the proposed model.
\end{abstract}

Index Terms-Wind power generation, power generation dispatch, operating reserve, expected energy not served, loss of load probability, wind incurred system cost, Q-function approximation.

\section{NOMENCLATURES}

$\begin{array}{ll}w & \text { Actual output energy (MWh) of wind turbine } \\ w_{f} & \text { Forecast output energy (MWh) of wind turbine } \\ \varepsilon_{w} & \text { Forecast error of wind turbine } \\ P W & \text { Actual output energy (MWh) of wind farm } \\ P W_{f} & \text { Forecast output energy (MWh) of wind farm } \\ \varepsilon_{w f} & \text { Forecast error of wind farm } \\ \eta_{f} & \text { Ratio term of maximal capacity by forecast output in (3) } \\ \hat{\varepsilon}_{w f} & \text { Forecast error of wind farm in \% }\end{array}$

This work was supported by the HKU Seed Funding Program for Basic Research (Project code: 200811159092), HKU Strategic Research Theme and the University Development Funding (Initiative on Clean Energy \& Environment).

K. Liu and J. Zhong are with the department of electrical and electric engineering, The University of Hong Kong, Hong Kong (e-mail: kailiu@eee.hku.hk)

\begin{tabular}{|c|c|}
\hline$\rho$ & Correlation coefficients matrix \\
\hline$\rho_{i j}$ & Correlation of forecast errors between two wind farms \\
\hline$W P$ & Actual output energy of total wind power in system \\
\hline$W P_{f}$ & Forecast output energy of total wind power in system \\
\hline$\varepsilon_{w p}$ & Forecast error of total wind power in system \\
\hline$\sigma_{w p}$ & Standard deviation of total wind power forecast error \\
\hline$L$ & Actual system load \\
\hline$L_{f}$ & Forecast system load \\
\hline$\varepsilon_{L}$ & Forecast error of system load \\
\hline$D$ & Actual net demand \\
\hline$D_{f}$ & Forecast net demand \\
\hline$\varepsilon_{d}$ & Forecast error of net demand \\
\hline$\sigma_{\mathrm{d}}$ & Standard deviation of forecast net demand error \\
\hline$L O L P$ & Loss of Load Probability \\
\hline EENS & Excepted Energy Not Served \\
\hline$V O L L$ & Value of Lost load \\
\hline$Q(\cdot)$ & Q-function \\
\hline$i$ & Index of conventional power generation unit \\
\hline$j$ & Index of wind farm in dispatch \\
\hline$n$ & Index of node bus \\
\hline$k$ & Index of possible congested transmission line \\
\hline$P_{i}$ & Output of conventional power generation unit $i$ \\
\hline$R_{i}$ & SR provided by unit $i$, elements of vector $\mathbf{R}$ \\
\hline$P W_{j}$ & Dispatched output of wind farm $j$, elements of $\mathbf{P W}$ \\
\hline$L_{n}$ & Load on bus $n$ \\
\hline$C_{i}(\cdot)$ & Offer energy cost function of unit $i$ \\
\hline$C R_{i}(\cdot)$ & Offer reserve cost function of unit $i$ \\
\hline$P T D F$ & Power Transfer Distribution Factors \\
\hline Iflow $w_{\max }^{k}$ & Capacity limit of transmission line $k$ \\
\hline$S$ & Upper bound of system wide LOLP index \\
\hline$P_{i}^{\max }$ & Capacity limit of unit $i$ \\
\hline$R C_{i}$ & Ramping capability limit for reserve of unit $i$ \\
\hline$W F_{j}^{\max }$ & $\begin{array}{l}\text { Maximal output of wind farm } j \text { available for dispatch } \\
\text { based on forecast output of wind turbines }\end{array}$ \\
\hline$\lambda$ & Lagrangian multiplier of constraint in (21.2) \\
\hline$\mu$ & Lagrangian multipliers of constraint in (21.3) \\
\hline$\alpha$ & Lagrangian multipliers of constraint in (21.4) \\
\hline$\beta$ & Lagrangian multipliers of constraint in (21.5) \\
\hline$\gamma$ & Lagrangian multipliers of constraint in (21.6) \\
\hline$\delta p$ & Lagrangian multipliers of constraint in (21.7) \\
\hline
\end{tabular}


$\delta r \quad$ Lagrangian multipliers of constraint in (21.8)

$\delta w \quad$ Lagrangian multipliers of constraint in (21.9)

$\tau \quad$ Lagrangian multipliers of constraint in (21.10)

\section{INTRODUCTION}

$\mathrm{N}^{\mathrm{o}}$ OWADAYS the world is facing the problem of global warning and energy crisis. Power generation sector contributes more than $30 \%$ of carbon dioxide emissions in the world and the collective efforts are needed from the power sector for this global warming issue. It is a trend to use clean energy (renewable energy), including wind, solar, hydro tidal and biology etc, to replace conventional fuel generations in the future. U.S. department of energy (DOE) has investigated the feasibility of supplying $20 \%$ of national demand by wind energy by 2030 [1]. North American Electric Reliability Corporation (NERC) also published the report to review the challenges of integrating high levels of variable generations into bulk American power system [2]. European Union targets at reducing greenhouse gas emission by $20 \%$, establishing a $20 \%$ share of renewable energy and reducing the overall energy consumption by $20 \%$, which is $20 / 20 / 20$ target by 2030 [3]. Since the large penetrations of intermittent renewable energy sources, such as wind power, will greatly impact the conventional operation of power system, integrating high level of intermitted renewable energy is also one of the major tasks of the next generations of power grid, the smart grid [4].

Beyond the economic and environmental benefits of renewable energy, the reliability of supplying all loads must be highlighted first. Power system reliability is concerned with possibility for the risk of not having sufficient generation capacity to serve all loads and it is measured in terms of the largest contingency, loss of load probability (LOLP), loss of load expectation (LOLE), expected energy not served (EENS), reserve at risk ( $\mathrm{RaR})$ [5], etc. Such risks are associated with the uncertainties in the system operation, such as largest generating unit outage, unforeseen increase in load, transmission line outage due to bad weather or the combination of these cases, etc. It is important to maintain the minimal amount of operating reserve to lower the risk at an accepted level, or say to run the power system at a secure manner. Obviously, the requirement of reserve highly depends on the uncertainties in the system, and the cost of such reserve resource is far from negligible.

The variable outputs of renewable energy generations significantly increase the uncertainty in system operation. It is not an easy task for system operators to match the supply and demand while maintaining operation criteria such as frequency and voltage within limits. Intuitively the operating reserve requirement will increase in accordance with penetration of intermittent renewable energy source. Therefore some issues related to market operation and system dispatching are rising. For example, connecting a large portion of wind power to a system with short or expensive resources of spinning reserves (SR) may not be practical or economic. It is desired to determine the optimal SR by integrated amounts of wind power for the power system, associated with the most economical and reliable system operation manner [6]-[8].

This paper will investigate the timely issue of scheduling variable generations of renewable energy in a market operation framework. Integrating renewable energy can impact both energy market and reserve market. Therefore the co-optimization for energy and reserve markets is used in this paper. The risk contributed by connecting renewable source is valued by predefined cost of loss function, with value of lost load (VOLL) and EENS. The problem is solved in the optimal power flow (OPF) framework, and the methodology is easy to convey to the traditional economic dispatch scenarios. The variation of total renewable energy sources in the system is modeled by statistical forecast error with Gaussian distribution. The wind power is used in the model as the type of renewable energy for its widely application and adequate data for simulation. Since the methodology is to resolve the system operation problem, the probabilistic solution methods, such as Monte Carlo simulation and heuristic methods, have the shortage of low computation speed. Thus we use Q-function to approximate the renewable energy contributed risk indices so that they become explicit and differentiable expressions and easy to solve in the optimization model.

This paper is organized as follows. In Section II, the wind power contributed reliability indices LOLP and EENS, are formulated. These indices are also related to the level of spinning reserves. The approximations are derived for sensitivity analysis and further optimization. In Section III the optimization model for coordinating wind power, traditional generations and cost of load loss is proposed and solved. In Section IV numerical simulation result are given based on the IEEE 39-bus system to show validities of proposed model and methodology. Section $\mathrm{V}$ is the conclusions.

\section{WIND POWER CONTRIBUTED LOLP AND EENS}

\section{A. Forecast Error of Wind Power Output}

With large penetration of wind power capacity, forecasting outputs of wind turbines and their error are very important for system operators. Wind power forecasting models provide the probabilistic estimation of wind turbines. The system operator can use the expected outputs of wind generations for generating units scheduling, such as unit commitment (UC) and economic dispatch (ED), and use the forecast errors of wind turbines to arrange operating reserves.

As a common practice in the literature, the forecast error of a wind turbine is viewed as a statistical error. Many researches have shown that the standard deviation of forecast error is against the forecast horizon. The actual output of a wind turbine can be assumed to be equal to the forecast value plus a statistical forecast error.

$$
w=w_{f}+\varepsilon_{w}
$$

Now considering the wind farm with many installed wind turbines with similar sizes, the forecast error of hourly output of 
each wind turbine is completely correlated for their same geographical locations. Thus the forecast error of whole wind farm can be the algebraic summation of errors of the connected wind turbines. The actual output of a wind farm can be also formulated as the forecast value plus a statistical forecast error, with respect to the individual forecast parameter $w_{f}$ and statistic error $\varepsilon_{w}$ in (1).

$$
\begin{aligned}
P W & =P W_{f}+\varepsilon_{w f} \\
\varepsilon_{w f} & =\frac{P W_{f}}{w_{f}} \varepsilon_{w} \\
& =P W_{f} \frac{W F_{\max }}{w_{f}} \frac{\varepsilon_{w}}{W F_{\max }}=P W_{f} \cdot \eta_{f} \hat{\varepsilon}_{w f}
\end{aligned}
$$

The overall forecast error of total wind power in the system can be modeled as Gaussian distribution. The standard derivation of this total forecast error needs to concern the variances and correlation coefficients of each wind farm. Such correlation of two wind farms decreases as the distance between them increases, which is shown in Fig.1 from [9]. With statistics data of wind farms in the system, a correlation matrix can be drawn to formulate the overall error of wind power. Therefore the forecast error of overall wind power in the system with the normal distribution can be formulated as follows.

$$
\begin{gathered}
\rho=\left[\rho_{i j}\right]_{\mathrm{N} \times \mathrm{N}} \\
W P=W P_{f}+\varepsilon_{w p} \\
\sigma_{w p}=\sigma\left(\varepsilon_{w p}\right)=\sqrt{\sum_{i} \sigma\left(\varepsilon_{w f, i}\right)^{2}+\sum_{j \neq i} \sum_{i} \sigma\left(\varepsilon_{w f, i}\right) \cdot \sigma\left(\varepsilon_{w f, j}\right) \cdot \rho_{i, j}}
\end{gathered}
$$

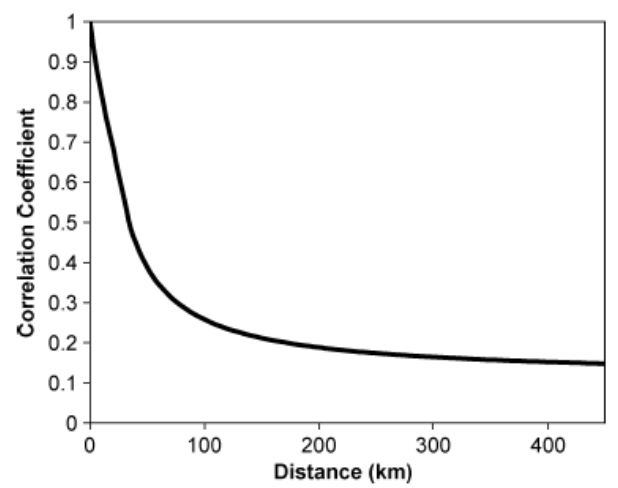

Fig. 1. Correlation coefficient between forecast errors of two wind farms' versus their distances

\section{B. Net Demand}

The actual system load is stochastic and it can be formulated as the forecast load plus a normal distributed forecast error.

$$
L=L_{f}+\varepsilon_{L}
$$

The net demand is defined as the system load minus the overall wind power output in the system and needs to be balance by conventional units in the system.

$$
\begin{aligned}
D & =L-W P \\
& =L_{f}-W P_{f}+\varepsilon_{L}-\varepsilon_{w p}=D_{f}+\varepsilon_{d}
\end{aligned}
$$

The net load is the sum of two stochastic values, load and wind power. In a common sense, the load and wind power can be assumed to be uncorrelated. Such case may be also altered with increased distributed renewable generation deployed in demand side in the future. In this paper, with separated load and wind power, the error of forecast net demand follows Gaussian distribution and is given as follows.

$$
\sigma_{\mathrm{d}}=\sigma\left(\varepsilon_{\mathrm{d}}\right)=\sqrt{\sigma\left(\varepsilon_{L}\right)^{2}+\sigma\left(\varepsilon_{w p}\right)^{2}}
$$

\section{Formulation of Wind Power Contributed LOLP/EENS}

Intuitively, increased penetration of wind power generation will deteriorate reliability index of the system. Wind power increases forecast error of net demand from (9) and such error is far from negligible. The net demand should not exceed the capacity of scheduled generating units $Q$ plus amount of total spinning reserve $R$ in the system to avoid undesired load shedding.

$$
\begin{aligned}
& \left\{\begin{array}{l}
D=L-W P+\sigma_{d} \geq Q+R \\
L-W P=Q
\end{array}\right. \\
& \Rightarrow \sigma_{d} \geq R
\end{aligned}
$$

The equation (11) is the power balance equation. The LOLP is the cumulative probability that the net demand error $\sigma_{d}$ is larger than SR. The EENS is the expect load shedding while $\sigma_{d}$ is larger than SR. By synthesizing (10) and (11), the expressions of LOLP and EENS are given respectively in follows.

$$
\begin{aligned}
& L O L P=\operatorname{Prob}\left(\sigma_{d}>R\right) \\
& E E N S=E\left\{\sigma_{d}-R \mid \sigma_{d}>R\right\}
\end{aligned}
$$

Since the error of the net demand is modeled as Gaussian distribution with standard derivation $\sigma_{d}$, the details of (12) and (13) are presented in follows.

$$
\begin{aligned}
\operatorname{LOLP} & =\int_{\mathrm{R}}^{+\infty} \frac{1}{\sqrt{2 \pi} \sigma_{\mathrm{d}}} \exp \left\{-\frac{x^{2}}{2 \sigma_{\mathrm{d}}{ }^{2}}\right\} d x \\
& =\operatorname{erfc}\left(\frac{\mathrm{R}}{\sqrt{2} \sigma_{\mathrm{d}}}\right)
\end{aligned}
$$




$$
\begin{aligned}
\text { EENS } & =\int_{\mathrm{R}}^{+\infty} \frac{x-R}{\sqrt{2 \pi} \sigma_{\mathrm{d}}} \exp \left\{-\frac{x^{2}}{2 \sigma_{\mathrm{d}}^{2}}\right\} d x \\
= & \int_{\mathrm{R}}^{+\infty} \frac{x}{\sqrt{2 \pi} \sigma_{\mathrm{d}}} \exp \left\{-\frac{x^{2}}{2 \sigma_{\mathrm{d}}^{2}}\right\} d x-R \int_{\mathrm{R}}^{+\infty} \frac{1}{\sqrt{2 \pi} \sigma_{\mathrm{d}}} \exp \left\{-\frac{x^{2}}{2 \sigma_{\mathrm{d}}^{2}}\right\} d x \\
= & \frac{4 \sigma_{\mathrm{d}}}{\sqrt{2 \pi}} \exp \left\{-\frac{R^{2}}{2 \sigma_{\mathrm{d}}{ }^{2}}\right\}-\operatorname{Rerfc}\left(\frac{\mathrm{R}}{\sqrt{2} \sigma_{\mathrm{d}}}\right) \\
\operatorname{erfc}(\mathrm{Z}) & =\int_{\mathrm{Z}}^{\infty} \frac{1}{\sqrt{\pi}} \exp \left\{-x^{2}\right\} \mathrm{dx}
\end{aligned}
$$

The error function in (16) is a complex function and can not be solved directly. The proper approximation is desired for the expression of LOLP and EENS in (14) and (15) for fast computation and implementation in further optimization model. Therefore we use Q-function to approximate the error function of Gaussian distribution.

$$
\begin{aligned}
Q(Z) & =\frac{1}{\sqrt{2 \pi} \mathrm{Z}} \exp \left\{-\frac{\mathrm{Z}^{2}}{2}\right\} \\
& \approx \int_{\mathrm{Z}}^{\infty} \frac{1}{1.94 \sqrt{2 \pi}} \exp \left\{-\frac{x^{2}}{2}\right\} d x
\end{aligned}
$$

$$
Q(Z) \geq \operatorname{erfc}(Z)
$$

The Q-function in (17) is the upper limit of the error function in (16). This explicit Q-function is a good approximation for the error function and with the considerable feature that the accuracy of approximation increases sharply while $\mathrm{Z}$ becomes large [10]. The Q-function approximation and the true value for the standard Gaussian distribution is compared in Table I and Fig. 2 as follows.

TABLE I

Comparison of the Q-function approximation and true value for standard normal distribution

\begin{tabular}{c|c|c|c}
\hline \hline $\mathrm{Z}$ & True value & $\begin{array}{c}\text { Approximation } \\
\text { value }\end{array}$ & Error in \% \\
\hline 2 & 0.02275 & 0.02466 & -8.41 \\
3 & 0.001350 & 0.001350 & 0.0148 \\
3.3 & 0.0004834 & 0.0004769 & 1.35 \\
3.5 & 0.0002326 & 0.0002278 & 2.07 \\
3.7 & 0.0001078 & 0.0001049 & 2.70 \\
4 & 0.00003167 & 0.00003057 & 3.48 \\
4.5 & 0.000003398 & 0.000003245 & 4.49 \\
\hline
\end{tabular}

The approximation in (17) is suitable for modeling reliability problem of power system. Power industry always needs to maintain a high standard of reliability. As industry standard "one day in ten years", it is a small number and in term of probability, it is in the order of $10^{-4}$. This reliability standard falls outside 3.5 times of standard derivation. From the comparisons above, the error offset of approximation can be less than $10^{-5}$ which is relative small. Such approximation can fasten the computation of reliability and easy the optimization model while maintaining the accuracy of result.

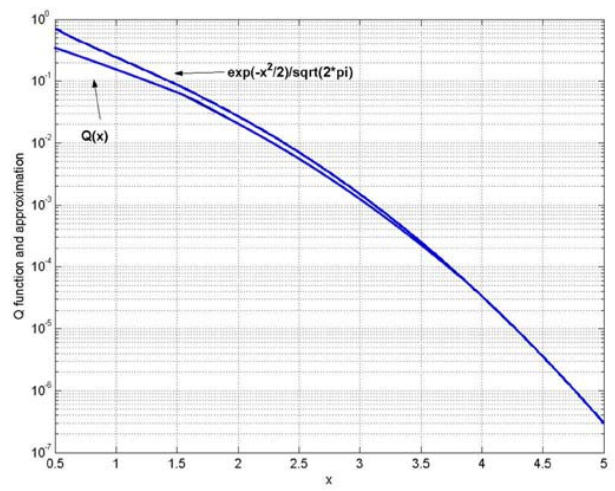

Fig. 2. Q-function approximation for standard normal distribution in lognormal plots

With the approximation in (17), expression of EENS and LOLP can be explicit given as follows with parameters from (1) to (18).

$$
\begin{aligned}
& \text { LOLP }=\frac{\sigma_{\mathrm{d}}}{1.94 \sqrt{2 \pi} R} \exp \left\{-\frac{R^{2}}{2 \sigma_{\mathrm{d}}{ }^{2}}\right\} \\
& \text { EENS }=\frac{3.49 \sigma_{\mathrm{d}}}{\sqrt{2 \pi}} \exp \left\{-\frac{R^{2}}{2 \sigma_{\mathrm{d}}{ }^{2}}\right\}
\end{aligned}
$$

The value of $\sigma_{d}$ can be solved from (1)-(9). Thus, the LOLP and EENS contributed by stochastic output of wind generation can be computed very fast without Monte Carlo simulation. The expressions (19) and (20) can maintain high accuracy while reliability standard of system is high, such as LOLP $>99 \%$, which is a normal case for power system. Beside the high accuracy, the approximation is with good mathematical properties such as smooth and differential. Such properties greatly facilitate the optimization model in next section.

\section{CO-OPTIMIZATION MODEL WITH WIND FARMS, OPERATING RESERVE AND RELIABILITY COST}

With operating costs nearly zero, the wind farms are usually pre-dispatched before the UC/ED procedures of market operation for other conventional generating units for the system with limited wind power capacity. However the situation will change with large penetrations of wind power in total system generation capacity, such as more than $30 \%$ wind power in system capacity. The stochastic output of wind generation can greatly increase the error of net demand in (9) and violate LOLP in (19) and EENS in (20). To maintain the reliable supply for the load, the system should increase the operating reserve capacity by means such as procurement from ancillary service market and synchronizing back up generating units. 
The wind generations can lower the cost by saving in energy market, and simultaneously they increase the reliability cost of system and cost of operating reserve.

One alternative is to coordinate the wind generation with additional operating reserve to maintain reliability standard higher than a desired level. This equals to predetermine the required capacity of operating reserve with assumption that demand of operating reserve is inelastic. With the development of smart grid technologies, such as AMI and energy storage, demand side of power system will become more and more flexible. It is desired to involve reliability index (or interruption cost) into the model of operating reserve. However such kinds of operating reserve models are mostly probabilistic models and complicated to solve.

For future power system with a large penetration of wind generations, the operator may need to analyze the wind power, operating reserve and reliability index in a unified framework. In a market structure, wind power impacts energy market and the operating reserve resource is procured from reserve market. Therefore this paper attempts to co-optimize the energy market including wind generations, reserve market and reliability cost simultaneously with proposed EENS and LOLP in (19) and (20). For simplicity, the interruption cost caused by conventional generation outage is omitted from calculation of reliability index.

The model pursues an optimal solution for conventional power generation units, renewable energy units, operating reserve and reliability cost all together. Energy and reserve are co-optimized in a market clearing process. Reliability cost is measured by value of lost load (VOLL) multiple by EENS. For simplification, only spinning reserve is considered in this paper. The objective function is formulated as follows.

$$
\min \left\{\sum_{i} C_{i}\left(P_{i}\right)+\sum_{i} C R_{i}\left(R_{i}\right)+\operatorname{EENS}(\mathbf{R}, \mathbf{P W}) \times V O L L\right\}
$$

The market structure is assumed to be a pool. The first term of objective function (21.1) is the total offered costs of conventional generating units. Vector $\mathbf{P W}$ is consisted of the dispatched outputs of each wind farm. The outputs of wind farm are adjusted by controlling the number of connected wind turbines with individual forecasted output. Here the wind farm outputs are modeled as continuous variables for simplification. The neglectable operating cost of wind farm is not considered here but the model can allow the wind farm bids in the energy market by adding terms in objective function. The second term of objective function is the total offered cost of provided reserve from conventional generating units. The third term of objective function is the expression of reliability cost accounting for the cost of the risk of load loss caused by the stochastic output of wind generations. VOLL is defined as the value an average consumer puts on an unsupplied MWh of energy [12]. The expression of EENS has been given in (1)-(9) and (20).

The objective function is subject to the following constraints:
1)Energy balance constraints

$$
(\lambda): \quad \sum_{i} P_{i}+\sum_{j} P W_{j}=\sum_{n} L_{n}
$$

The energy balance constraint ensures that the total supply of generating units and total forecast output of wind generations can meet the total demand in (21.2). The system loss is not considered here for simplicity.

2) Transmission constraints

$$
\begin{gathered}
\left(\mu_{\mathrm{k}}\right): \sum_{i} P T D F_{i}^{k} \cdot P_{i}+\sum_{j} P T D F_{j}^{k} \cdot P W_{j}-\sum_{n} P T D F_{n}^{k} \cdot L_{n} \\
\leq I f l o w_{\max }^{k} \text { for any congested line } k
\end{gathered}
$$

The DC power flow with Power Transfer Distribution Factors (PTDF) is used to represent the transmission flow by each individual resource's out in this paper.

3)Lower limit of system wide LOLP:

$$
(\alpha): \quad \operatorname{LOLP}(\mathbf{R}, \mathbf{P W}) \geq S
$$

From engineering views, it is assumed that the probability of losing load should be under a certain level, such as $5-10 \%$ which is much higher than the current industry reliability standard. This constraint can also use other indices, such as EENS, RaR [5] or the largest on line generating units etc. The constraint in (21.4) also ensures that approximations in (19) and (20) are accurate enough for the model.

4)Capacity constraints for generation units

$$
\left(\beta_{\mathrm{i}}\right): \quad P_{i}+R_{i} \leq P_{i}^{\max } \quad \text { for any } i
$$

5)Ramp capacity constraints for reserve units

$$
\left(\gamma_{\mathrm{i}}\right): \quad R_{i} \leq R C_{i} \quad \text { for any } i
$$

6)Bounds of control variables

$$
\begin{aligned}
& \left(\delta p_{i}\right): \quad P_{i} \geq 0 \text { for any } i \\
& \left(\delta r_{i}\right): \quad R_{i} \geq 0 \text { for any } i \\
& \left(\delta w_{j}\right): \quad P W_{j} \geq 0 \text { for any } j \\
& \left(\tau_{j}\right): \quad P W_{j} \leq W F_{j}^{\max } \text { for any } j
\end{aligned}
$$

The upper limits of wind farm output $W F_{j}^{\max }$ are the maximal outputs with total wind turbines available for dispatching based on their forecast outputs. In addition the reserve from offline units is not concern for simplicity.

The co-optimization model has been given in above formulations. With (1) to (20), all the expressions in model are analytical and explicit, and available to be solved by existing 
nonlinear optimization solvers such as MINOS [11].

Otherwise with proposed formulation and optimization model, the marginal system costs of wind energy can be easily calculated according to Karush-Kuhn-Tucker (KKT) condition. Such marginal cost index can help independent system operator (ISO) to dispatch according to the incremental costs by additional wind generations and allocate such costs to each wind farms fairly.

\section{Simulation Results}

In this section, we will present the case studies of proposed co-optimization model based on the IEEE-39 bus system. The optimization of simulation is solved by software platform GAMS. In the example, 9 thermal generators and 6 wind farms participate in the energy and reserve markets. Generators G3 and G4 have no capability of spinning reserve. The forecast and error data of wind turbines in wind farms are collected from U.S. eastern interconnection. The value of VOLL is chose from 1000 to $8000 \mathrm{USD} / \mathrm{MWh}$ in the case study. Considering EENS/LOLP are contributed only by wind energy, generator outages are not concerned here for simplicity.

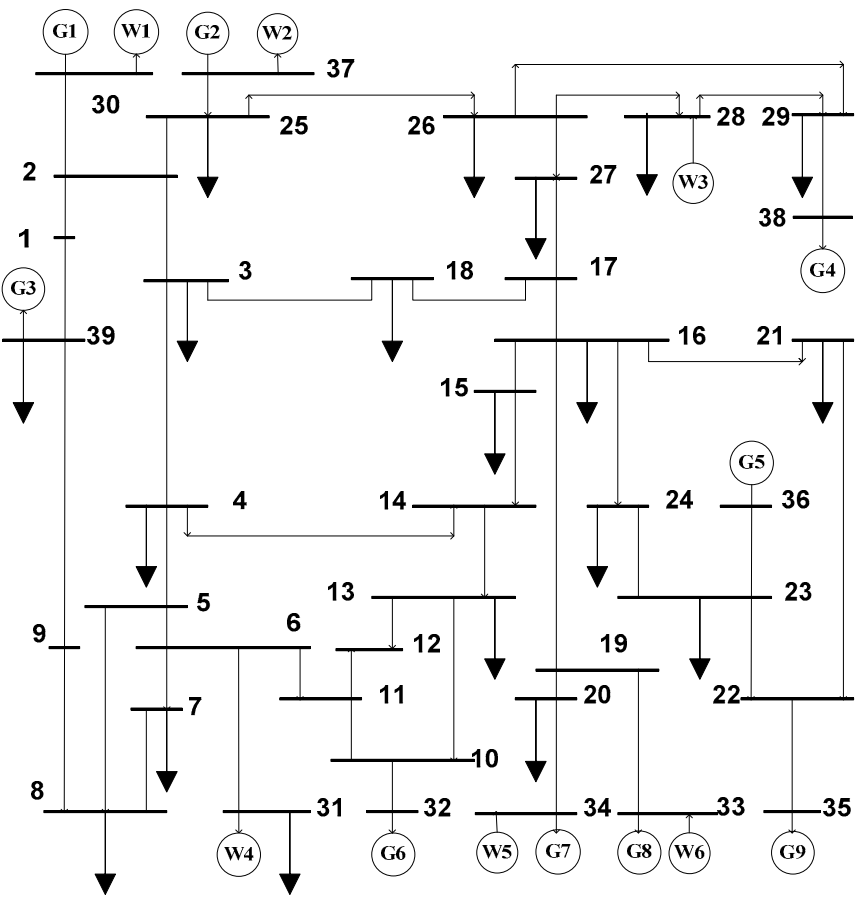

Fig. 3. IEEE-39 bus system with generating units in test

The results of the co-optimization are given in Table II, where VOLL is $4000 \mathrm{USD} / \mathrm{MWh}$. The computation time by GAMS is 0.001 seconds. The objective value, total hourly cost in (21), is 308,188 USD. The system LOLP and EENS are $0.0167 \%$ and 1.701 respectively.
TABLE II

Results of co-optimization model

\begin{tabular}{c|c|c|c}
\hline \hline $\begin{array}{c}\text { Units } \\
\text { number }\end{array}$ & $\begin{array}{c}\text { Energy } \\
\text { (MWh) }\end{array}$ & $\begin{array}{c}\text { Reserv } \\
\text { e } \\
\text { (MWh) }\end{array}$ & $\begin{array}{c}\text { Maximal } \\
\text { outputs (MWh) }\end{array}$ \\
\hline G1 & 233.7 & 103.8 & 337.5 \\
G2 & 485.5 & 243.5 & 729 \\
G3 & 1170 & 0 & 1350 \\
G4 & 922.1 & 0 & 1120.5 \\
G5 & 533.8 & 222.2 & 756 \\
G6 & 623.9 & 253.6 & 877.5 \\
G7 & 490.0 & 195.8 & 685.8 \\
G8 & 618.8 & 234.4 & 853.2 \\
G9 & 661.6 & 215.9 & 877.5 \\
\hline Wind farm & \multicolumn{2}{|r}{} & WF \\
\hline W1 & 303.3 & & 350 \\
W2 & 300.0 & & 300 \\
W3 & 310.0 & & 310 \\
W4 & 400.0 & & 400 \\
W5 & 340.0 & & 340 \\
W6 & 299.9 & & 540 \\
\hline
\end{tabular}

The dispatch results of wind farms with different values of VOLLs are presented in Table 3. The outputs of wind farms in optimal solution of system decease with increased VOLL. While the VOLL is small, such as $1000 \mathrm{USD} / \mathrm{MWh}$ in the second column, wind farms $\mathrm{W} 1$ to $\mathrm{W} 5$ are arranged to output at their maximal levels. For these wind farms in this case, the marginal incurred reliability costs are lower than the marginal deceased costs in energy market. For W6, the system is inefficient to add reserve to support its increasing output. Otherwise it can be observed that only $\mathrm{W} 1$ and $\mathrm{W} 6$ are decreasing with increased VOLL. It is because that the output value $W_{j}$ weighs slightly in the sensitivity of wind cost in (27). Therefore for the practical market operation, the outputs of wind farms may need to be re-allocated to balance the revenue for wind farms with various allocation and forecast tools.

TABLE III

Results of wind farms with various VOLLs

\begin{tabular}{c|c|c|c|c}
\hline \hline \multirow{2}{*}{$\begin{array}{l}\text { WF } \\
\text { max }\end{array}$} & \multicolumn{4}{|c}{ VOLL (USD/MWh) } \\
\cline { 2 - 5 } MWh) & 1000 & 2500 & 4800 & 8000 \\
\hline $\mathrm{W} 1=350$ & 350.0 & 350.0 & 287.1 & 247.1 \\
$\mathrm{~W} 2=300$ & 300.0 & 300.0 & 300.0 & 300.0 \\
$\mathrm{~W} 3=310$ & 310.0 & 310.0 & 310.0 & 310.0 \\
$\mathrm{~W} 4=400$ & 400.0 & 400.0 & 400.0 & 400.0 \\
$\mathrm{~W} 5=340$ & 340.0 & 340.0 & 340.0 & 340.0 \\
$\mathrm{~W} 6=540$ & 454.5 & 334.8 & 268.2 & 226.5 \\
\hline
\end{tabular}

\section{CONCLUSIONS}

This paper presents an analytical framework for the dispatch problem of power system with significant installed capacity of wind generations. The reliability indices, EENS and LOLP, contributed by stochastic outputs of wind generation are 
modeled and formulated. The Q-function approximation is used for deriving the explicit expressions of index formulations. A co-optimization model of energy and reserve market with consideration of reliability cost by EENS is presented to study the optimal allocation of wind power, reserve and reliability cost. The proposed model is deterministic and analytical. An equivalent marginal wind power incurred cost is proposed and given in (26). Such equivalent wind costs can be used to allocate the incremental system cost with respect to reserve and reliability (interruption) cost to the dispatched wind generations. The simulation test based on IEEE-39 bus system is carried out to prove the validities of proposed model and formulation. The model can be easily implemented and solved very fast with the now available optimization software, such as GAMS. The future works include elaborating the model and formulation with truncated distribution of wind forecast errors. Under the assumption that the forecast error of overall wind power is normal distribution, the analysis framework in this paper can work with considerations of altered means and standard deviations by truanted distribution for forecast error of individual wind generations.

\section{REFERENCES}

[1] DOE Report, “20\% Wind Energy by 2030: Increasing Wind Energy's Contribution to U.S. Electricity Supply", available: http://www1.eere.energy.gov/windandhydro/wind_2030.html, July 2008.

[2] NERC report "Accommodating High Levels of Variable Generation", available: http://www.nerc.com/files/IVGTF_Report 041609.pdf

[3] "Climate change: Commission welcomes final adoption of Europe's climate and energy package," Press Release, EU, available: http://europa.eu/rapid/pressReleasesAction.do?reference=IP/08/1998

[4] EPRI Smart Grid, available: http://www.smartgrid.epri.com/

[5] F.F. Wu, H. Zhou, Y. H. Hou, "Measuring Reliability (or risk) Coherently", in Proc. PSCC July 14-18, 2008 Glasgow, Scotland,

[6] L. Soder, "Reserve margin planning in a wind-hydro-thermal power system," IEEE Trans. on Power Systems, vol. 8, pp. 564-571, 1993.

[7] R. Doherty and M. O'Malley, "A new approach to quantify reserve demand in systems with significant installed wind capacity," Power Systems, IEEE Transactions on, vol. 20, pp. 587-595, 2005.

[8] M. A. Ortega-Vazquez and D. S. Kirschen, "Estimating the Spinning Reserve Requirements in Systems With Significant Wind Power Generation Penetration," IEEE Trans. on Power Systems, vol. 24, pp. 114-124, 2009.

[9] R. Watson and L. Landberg, "Evaluation of prediktor wind power forecasting system in Ireland," in Proc. Madrid Eur. Wind Energy Assoc. Conf., Jun. 2003.

[10] M.K. Steven, Fundamentals of Statistical Processing: Estimation Theory, Prectice Hall, 1993.

[11] GAMS software, available: http://www.gams.com

[12] R. Billinton and R.N. Allan, Reliability Evaluation of Power System. New York: Plenum, 1996.

\section{BIOGRAPHIES}

Kai Liu (S’06) received the B.Sc. degree from Tsinghua University, Beijing, China, in 2005 and the MPhil degree from the University of Hong Kong, Hong Kong, in 2007, both in electrical engineering. Currently he is pursuing the $\mathrm{Ph} . \mathrm{D}$ degree in the University of Hong Kong. His research topic is power system planning, and smart grid.
Jin Zhong (S'00-M'04) received the B.Sc. degree from Tsinghua University, Beijing, China, in 1995, the M.Sc. degree from China Electric Power Research Institute, Beijing, in 1998, and the Ph.D degree from Chalmers University of Technology, Gothenburg, Sweden, in 2003. At present, she is an Assistant Professor in the Department of Electrical and Electronic Engineering of the University of Hong Kong. Her areas of interest are smart grids, electricity sector deregulation, ancillary service pricing, and power system planning. 